it exists) soon after coming into operation in 1981 (perhaps). It would produce a considerably higher collision rate than the equivalent European machine; and Fermilab physicists are hoping that this will help persuade Congress to provide the necessary \$26 to \$29 million.

The second proposal, devised by $\mathrm{Dr}$ Richter, is to create single collisions between individual pulses of electrons and positrons. Both would be produced on the SLAC linear accelerator, using techniques now being developed to increase the beam energy from around $25 \mathrm{GeV}$ to $50 \mathrm{GeV}$; they would then be made to circulate in opposite directions around a circular tunnel, and carefully aimed to collide with each other.

Since each pulse will only produce a single collision, the event rate will be much lower than for the constantly circulating pulses in storage rings at a comparable energy. Dr Richter proposes to make up for this, however, by tuning the two beams to collide at a centre of mass energy calculated to be equal to be the mass of the $Z^{\circ}$ particle, using the resonance at this energy to increase the luminosity.

If the $Z^{\circ}$ does exist, then the Single Pass Collider, as the project is known, would become a virtual factory of decay particles with an event-rate around $10^{6}$ a year, and the facility could be used by many different users. If the $Z^{\circ}$ does not exist, the event rate will be very much slower; "but the physics will be much more interesting, since it would mean a basic flaw in the theory", says Dr Richter.

The design would lack the power and versatility of LEP, scheduled to reach 100 $\mathrm{GeV}$ per beam, and to be built by the late 1980 s. But at a cost of $\$ 60$ million compared to LEP's $\$ 1000$ million - plus the fact that it could be in operation as early as 1984 , the proposal is attractive to US physicists. "It is an extremely clever idea, real 'Yankee ingenuity' "' says one.

It all comes down to money. Dr Richter has asked the DoE for $\$ 800,000$ to carry out a preliminary design study; and says that, if given the go-ahead by the department and Congress in the 1982 fiscal year budget to be prepared next summer, the collider could be completed in under three years.

Whether Congress will go along with either this or the Fermilab project remains the great unknown; and few are overoptimistic. Last week the DoE's High Energy Physics Advisory Panel (HEPAP) supported a proposal that its chairman, $\mathrm{Dr}$ Sidney Drell of SLAC, write a letter to the department expressing grave concern at the current funding position; and warning that if there was not an immediate infusion of extra funds - at least sufficient to restore the budget to the promised "floor" - the panel would consider withdrawing its endorsement of the national programme. Department officials at the meeting promised the physicists a sympathetic hearing; but no more than that.

\title{
NIH director unlikely to grant exemption from controls for DNA experiments
}

The director of the US National Institutes of Health, Dr Donald Fredrickson, seems unlikely to accept in full the recommendation of the Recombinant DNA Advisory Committee (RAC) that a large body of recombinant DNA experiments be exempted from the guidelines established by the NIH, following widespread criticism of the implications of such a move.

Sources within the NIH say that Dr Fredrickson will probably approve a significant reduction in the safety precautions needed to carry out most experiments using as host the disabled $\mathrm{K}-12$ strain of the bacterium Escherichia coli; these are said to account for between 80 and $85 \%$ of all experiments using recombinant DNA techniques.

But whereas the RAC, in a split 10 to 4 vote, decided at its last meeting in September to recommend exemption for the guidelines for such experiments - with merely the ruling that they should be registered with local biohazard committees, and should preferably be carried out at the minimal $\mathrm{Pl}$ physical containment level - Dr Fredrickson is likely to insist that the $\mathrm{Pl}$ conditions be enforced. This will include strict adherence to technician training requirements, and a ban on practices such as mouth-pipetting.

The NIH has had a flood of comments since the proposal to exempt the experiments was first put forward by RAC members Dr Allen Campbell and Dr Wallace Rowe at the committee's meeting in May. Many scientists wrote supporting the proposal, including in particular a petition signed by 183 scientists attending the Gordon conferences on nucleic acids and on biological regulatory mechanisms.

Others, however, have expressed concern about the implications of such a drastic move. Professor Roy Curtiss, of the department of microbiology at the University of Alabama in Birmingham, wrote to Dr Fredrickson criticising the proposed exemptions as premature, given

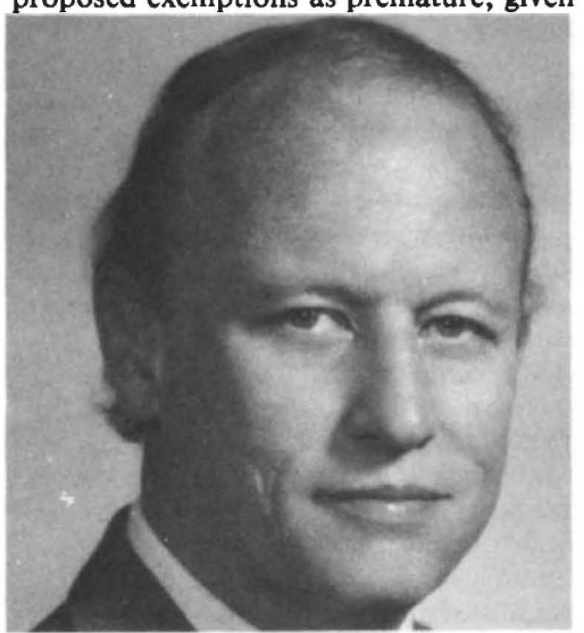

Fredrickson: reviewing the arguments the uncertainties that still exist over the hazards of recombinant organisms. "I believe that the RAC's recommendation to you was based more on the politics of science than on its data", he wrote.

In addition, the Natural Resources Defense Council in New York has demanded that an environmental impact statement be carried out for the proposed safeguard reductions, arguing that "the proposed exemptions are of such breadth and importance as to require full compliance with the National Environmental Policy Act.",

In view of the controversy surrounding the committee's recommendation and its implications, Dr Frederickson has been carrying out a detailed review both of the arguments used during the RAC meeting, and of the data used to support them.

The results of this review will not be known for several weeks. However it is thought that Dr Fredrickson is reluctant to face the criticism that total exemption would involve.

For example, two weeks ago the citizens of Amherst, which has introduced a local ordinance requiring recombinant DNA research at the University of Amherst to be conducted under the NIH guidelines, agreed to require adherence to the 1978 revision of the guidelines, rather than their stricter 1976 original version.

However according to Dr Bruce Levin, a research scientist at the university who has been closely involved in the local debates, there would probably be strong resistance from the local community if a large proportion of the experiments were to be exempt from regulation.

Dr Fredrickson, however, is said to be prepared to accept most of the arguments in favour of a substantial reduction in required containment levels for many experiments involving the $\mathrm{K}-12$ strain; one of the few areas still to be resolved is whether local committees would be required to give prior approval to experiments using biologically active materials, such as active polypeptides or active proteins.

The NIH is paying particular attention in its review to uncertainties that have arisen as a result of various risk assessment experiments, such as those which indicate that bacteria into which plasmids have been introduced can survive considerably longer than expected in the human gut.

Meanwhile staff members for Senator Adlai Stevenson's science and technology subcommittee are preparing legislation that would require all non-federally supported research involving recombinant DNA techniques - in particular that carried out by private companies - to register their experiments with the NIH. At present companies can register; but the arrangement is voluntary. 